\title{
La modélisation forestière au Québec : contexte, défis et perspectives ${ }^{1}$
}

\author{
by Guy R. Larocque ${ }^{2}$, Daniel Mailly ${ }^{3}$ et Mélanie Gaudreault ${ }^{3}$
}

\begin{abstract}
RÉSUMÉ
Lutilisation des modèles de productivité forestière est en constante augmentation pour la réalisation des calculs de possibilité forestière et pour la prise de décision en aménagement forestier au Québec. Un colloque a été organisé au printemps 2008 afin que modélisateurs, aménagistes, utilisateurs et gestionnaires puissent échanger sur le contexte, les défis et les perspectives d’avenir de la modélisation forestière. Suite à une série de conférences portant sur différents types de modèles et leur utilisation en aménagement forestier, les participants au colloque ont discuté de la méthodologie, de la collaboration et des orientations futures de la modélisation. Cet article présente les idées et suggestions recueillies lors des discussions. Celles-ci ont entre autres porté sur la nécessité de concevoir des modèles conviviaux, dont les limites d’utilisation sont connues, qui seront utilisés avec des données de qualité et pour lesquels il est possible de calculer le biais et lerreur de prévision. L'intégration de différents types de modèles ou de leurs prévisions est aussi une avenue à considérer. Afin de collaborer efficacement à l'avancement de la modélisation forestière, les concepteurs et les utilisateurs des modèles doivent former des équipes multidisciplinaires et développer des réseaux de communication efficaces afin de partager leurs connaissances en dehors des cercles habituels. Dans l'avenir, la mise au point de méthodes d’optimisation et de résolution de problèmes complexes et une plus grande utilisation des méthodes d'analyse de sensibilité et d'incertitude seront à privilégier. Enfin, une importance prépondérante devra dorénavant être accordée à des critères autres que la matière ligneuse, tels que la biodiversité, les habitats, les sols et l'impact des changements climatiques.
\end{abstract}

Mots-clés : Modélisation forestière; atelier; ACFAS

\section{ABSTRACT}

Forest productivity models are increasingly being used for the computation of allowable cuts and forest management decision-making in Quebec. A workshop was organized in the spring of 2008 to bring together modellers, managers, users and administrators to provide a forum for the exchange of views and opinions on the challenges and future perspectives in forest modelling. Following a series of oral presentations on the various types of models and their application in forest management, workshop participants held discussions on methodology, collaboration and future directions in modelling. The present article offers a summary of the ideas and suggestions generated during the discussions. Among other things, these dealt with the need to design user-friendly models, with known limitations, that could be used with good quality data and for which it would be possible to calculate the bias and forecasting error. Integrating different types of models or their forecasts is another avenue to consider. In order to effectively collaborate and contribute to the advancement of forest modelling, model developers and users must form multidisciplinary teams and develop efficient communication networks to share their knowledge outside of the usual circles. In the future, the development and adjustment of optimization and complex problem resolution methods as well as a greater use of sensitivity and uncertainty analytical methods

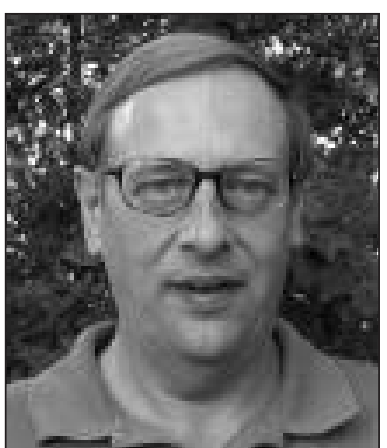

Guy R. Larocque

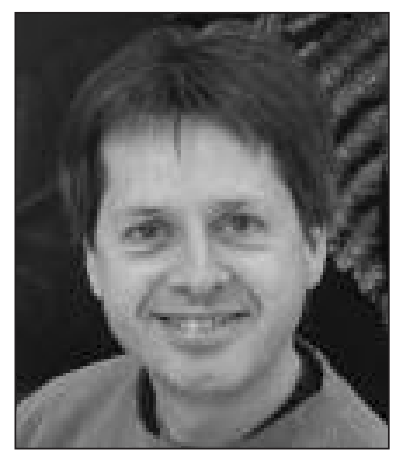

Daniel Mailly

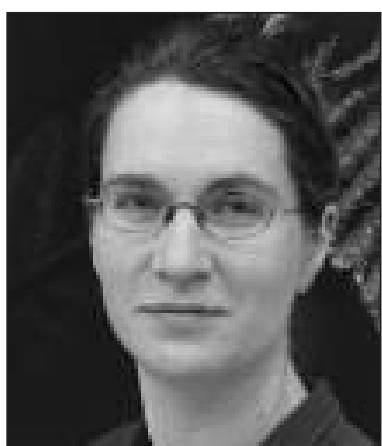

sized. Finally, much more importance should be given to criteria other than wood fibre, such as biodiversity, habitat, soils and the effect of climate change.

Key words: forest modelling, workshop, ACFAS

\footnotetext{
${ }^{1}$ Sommaire des présentations et discussions tenues lors du colloque « Comment concilier différentes approches de modélisation forestière dans une optique d’amélioration des outils d’aide à la décision à l’aménagement et des calculs de possibilitié? » ayant eu lieu lors du congrès annuel de l'Association francophone pour le savoir - ACFAS - à Québec, le 8 mai 2008.

${ }^{2}$ Ressources naturelles Canada, Service canadien des forêts, 1055, rue du P.E.P.S., C.P. 10380, Succ. Sainte-Foy, Québec (Québec) G1V 4 C7. Auteur à qui la correspondance doit être adressée. Courriel : Guy.LaRocque@RNCan-NRCan.gc.ca.

${ }^{3}$ Direction de la recherche forestière, Ministère des Ressources naturelles et de la Faune du Québec, 2700, rue Einstein, Québec (Québec) G1P 3W8
} 


\section{Introduction}

Les modèles de productivité forestière sont de plus en plus utilisés pour les calculs de possibilité forestière et la prise de décision en aménagement forestier. La pertinence de leur utilisation va en grandissant afin de répondre aux pressions du public concernant les enjeux environnementaux tels que lévolution de la biodiversité, l'impact des changements climatiques et l'application des principes d’aménagement forestier durable et écosystémique. La grande diversité des modèles existants rend toutefois la tâche plus difficile aux utilisateurs qui doivent sélectionner les outils appropriés pour répondre à leurs besoins. Un colloque sur la modélisation forestière a donc été organisé afin de permettre aux modélisateurs, aménagistes, utilisateurs et gestionnaires de faire le point sur le développement de la modélisation forestière et d'émettre leurs opinions et leurs besoins sur le sujet (Fig. 1). Ce colloque s'est tenu à Québec le 8 mai 2008, dans le cadre du congrès de l'Association francophone pour le savoir (ACFAS).

Une douzaine d’exposés portant sur différents types de modèles ont d'abord été présentés afin de montrer la diversité et létendue des possibilités offertes par les modèles de simulation forestière. Les conférenciers ont abordé les thèmes suivants : intégration des valeurs écologiques, forestières, économiques et sociales dans les modèles; développement de modèles régionaux et aménagement forestier; modélisation de la dynamique et du bilan de carbone; avancement des modèles dans le contexte québécois; réalisation de modèles par tiges individuelles; et application du concept de révolution écologique ${ }^{4}$. Des séances de discussion portant sur la méthodologie, la collaboration et les orientations futures de la modélisation forestière ont suivi. Les participants ont alors pris part aux discussions sur chacun de ces trois aspects. Dans cet article nous désirons présenter les idées et suggestions recueillies lors de ces séances.

\section{Méthodologie}

Au début des séances de discussion, les participants ont identifié des modèles appliqués à la foresterie qu'ils avaient déjà utilisés (Tableau 1). Vint ensuite la période de discussion sur des questions particulières. Les participants ont dabord discuté des caractéristiques les plus importantes d'un modèle devant servir doutil d’aide à la décision ${ }^{5}$. Les caractéristiques les plus fréquemment citées ont été la simplicité d'utilisation, la transparence, labsence de biais ou biais minimal, la généralité d’application, la connaissance de létendue d’application, de l'erreur et de l'incertitude et la disponibilité d'intrants mesurables avec le moins derreurs possible. Il a aussi été question de l'accès à des données de qualité pour réaliser lajustement des modèles et de la confiance que les utilisateurs avaient dans le modèle utilisé et les variables explicatives servant d'intrants. Lamélioration du processus décisionnel en aménagement forestier demande aussi la mise au point de méthodes d'intégration des prévisions de plusieurs modèles en tenant compte des connections essentielles entre les processus et les échelles spatiales et temporelles.

\footnotetext{
${ }^{4}$ Les présentations sont disponibles à l'adresse suivante : www. mrnf.gouv.qc.ca/externe/modelisation/.

${ }^{5}$ Le texte en italique fait référence aux questions posées aux participants lors des discussions.
}

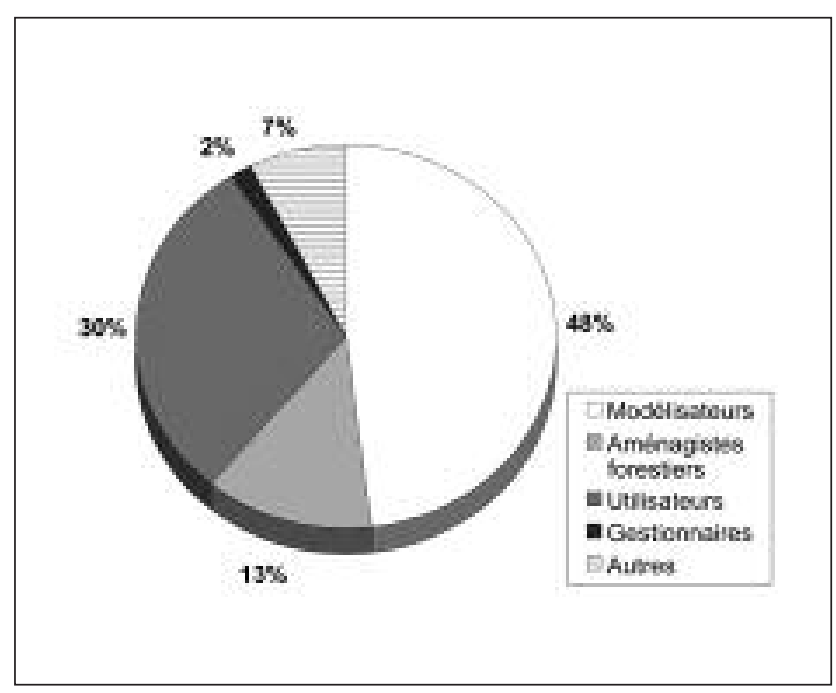

Fig. 1. Composition (\%) de l'assistance au colloque sur la modélisation forestière.

Plusieurs méthodologies ont ensuite été identifiées comme étant utiles afin d'intégrer les différentes approches de modélisation dans le but d'aider les aménagistes à prendre les meilleures décisions. La mise au point de logiciels facilement utilisables, flexibles et ayant une documentation bien structurée a été reconnue comme une mesure importante pour faciliter la tâche des usagers. L'intégration de différents types de modèles a aussi été proposée comme une approche à favoriser. À cet égard, le modèle TRIPLEX a été cité en exemple. L'intégration de différents types de modèles pourrait consister à combiner des modèles empiriques de croissance et des modèles fondés sur les processus (modèles hybrides) et devrait inclure des composantes qui permettent deffectuer des analyses de sensibilité et d'incertitude. Enfin, les langages de programmation orientés vers les objets devraient être privilégiés.

Parmi les points à retenir, les participants ont mentionné qu'il est essentiel de porter attention à la qualité des données, destimer adéquatement le biais et lerreur de prévision, de veiller à la simplicité d'utilisation, de demeurer à l'affût de ce qui se fait ailleurs, de s'assurer que la méthodologie demeure en lien avec l'objectif visé et de comparer la pertinence d'utiliser des modèles provinciaux par rapport aux modèles locaux.

\section{Collaboration}

Dans le cadre de ce thème, les participants de l'atelier ont été amenés à se prononcer sur les caractéristiques essentielles pour réussir une bonne collaboration. Selon la majorité d'entre eux, les qualités telles que la transparence, la communication et le respect constituent la base d'une bonne collaboration. Les participants tiennent aussi à former des équipes multidisciplinaires composées de personnes ayant des expertises complémentaires et qui trouvent chacune un avantage à participer au travail (situation gagnant - gagnant), tout en ayant une vision commune et des affinités personnelles. Finalement, les participants ont mentionné que la disponibilité de fonds de recherche et le partage des connaissances et des données constituent également des facteurs de réussite d’une bonne collaboration. L’ensemble de ces caractéristiques est jugé nécessaire 
Tableau 1. Liste de quelques modèles de simulation forestière que les participants ont affirmé avoir déjà utilisés

Modèles empiriques

Relations allométriques

Tables de production

Tables de production de Pothier et Savard

Tables de production de Boudoux

Modèles de calculs de possibilité forestière

SYLVA II

Modèles de croissance

Forest Vegetation Simulator (FVS)

Modèles de succession

SORTIE

ZELIG

Modèles fondés sur les processus

TRIPLEX
Lambert, M.-C., C.-H. Ung et F. Raulier. 2005. Canadian national tree aboveground biomass equations. Can. J. For. Res. 35 : 1996-2018.

Pothier, D. et F. Savard. 1998. Actualisation des tables de production pour les principales espèces forestières du Québec. Direction des inventaires forestiers, ministère des ressources naturelles du Québec. 183 p.

Boudoux, M. 1978. Tables de rendement empiriques pour l'épinette noire, le sapin baumier et le pin gris au Québec. Ministère des Terres et Forêts du Québec, Québec. COGEF. $101 \mathrm{p}$.

www.mrnf.gouv.qc.ca/produits-services/sylvaii

Stage, A.R. 1973. Prognosis model for stand development. USDA Forest Service, Intermountain Forest and Range Experiment Station, Research Paper INT-137. 32 p.

Teck, R., M. Moeur et B. Eav. 1996. Forecasting ecosystems with the Forest Vegetation Simulator. J. For. $94: 7-10$.

Pacala, S.W., C.D. Canham et J.A. Silander, Jr. 1993. Forest models defined by field measurements: I. The design of a northeastern forest simulator. Can. J. For. Res. 23 : $1980-1988$.

Urban, D.L. 1993. A user's guide to ZELIG version 2 with notes on upgrades from version 1. Department of Forest Sciences, Colorado State University, Fort Collins, CO, USA.

Peng, C., J. Liu, Q. Dang, M.J. Apps et H. Jiang. 2002. TRIPLEX: a generic hybrid model for predicting forest growth and carbon and nitrogen dynamics. Ecol. Model. 153: $109-130$. pour travailler d'une manière efficace et constructive à l'avancement de la modélisation forestière.

Une discussion a ensuite été engagée à propos de la meilleure forme de collaboration pour favoriser l'avancement des modèles de simulation forestière. Les participants ont d'abord mentionné l'importance de développer les relations et la communication entre les concepteurs et les utilisateurs des modèles. En effet, l'implication directe des utilisateurs dans le processus de conception des modèles ou par une rétroaction régulière est requise pour que les outils mis au point répondent aux besoins et soient utilisés à leur pleine mesure. Les participants ont également mentionné l'importance d'améliorer les liens entre les concepteurs des modèles pour favoriser les collaborations spontanées et le partage des connaissances. Afin de développer ces nouveaux canaux de communication, plusieurs participants ont suggéré l'ajout de moyens interactifs pour échanger et transmettre l'information plus rapidement et d'une manière plus étendue. Les tribunes identifiées comprennent des conférences réunissant les différents intervenants du milieu (les concepteurs, les utilisateurs et les décideurs), des forums de discussion, des blogues et des sites Internet conçus pour l'écriture collaborative (wiki).

Les participants ont aussi suggéré l'implantation d'un organisme indépendant qui aurait pour mandat de regrouper l'information sur les modèles disponibles et les travaux de recherche en cours au Québec ainsi que de vulgariser les résultats de recherche. Des organismes comme le Conseil de la recherche forestière du Québec (CRFQ) ou le Centre d'étude sur la forêt $\left(\mathrm{CEF}^{6}\right)$ pourraient se voir confier ce type de mandat. D'autres participants sont plutôt d'avis que des programmes de subvention de projets intégrateurs ou multidisciplinaires seraient la meilleure manière de favoriser les associations de gens provenant de différents milieux. La distribution de ces subventions pourrait, par exemple, être gérée par un organisme subventionnaire comme le Fonds québécois de la recherche sur la nature et les technologies

${ }^{6}$ Site du CEF : www.cef-cfr.ca. 
$\left(\mathrm{FQRNT}^{7}\right)$ ou prendre la forme de comités interministériels. Finalement, plusieurs personnes ont mentionné le besoin de créer des programmes de recherche à long terme afin d'assurer la stabilité du financement attribué à la modélisation et, du fait même, la continuité des collaborations.

En terminant, les participants ont mentionné que les ingrédients nécessaires à l'avancement de la modélisation forestière étaient présents, mais qu'il était primordial d'accroître la communication et la création des liens entre tous les intervenants, notamment entre les utilisateurs et les concepteurs des modèles.

\section{Orientations futures}

Lors des discussions sur ce thème, les participants se sont d’abord interrogés sur les lacunes actuelles au niveau des outils et approches de la modélisation appliquée à la foresterie. Des applications précises ont été mentionnées à plusieurs reprises et semblent avoir retenu plus particulièrement l'attention des participants. Par exemple, les aspects liés aux méthodes d’optimisation et de résolution de problèmes complexes et à l'analyse de sensibilité des résultats de simulation sont considérés comme étant de première importance. Ils ont été classés au même niveau que les considérations liées aux perturbations, aux sols et aux habitats, à la biodiversité ainsi quaux changements climatiques. Les aspects suivants ont également été reconnus comme importants : systèmes experts, succession forestière, spatialisation et localisation en lien avec le calcul de possibilité forestière, économie forestière, visualisation et modélisation à léchelle du paysage, modélisation de la qualité des tiges et des traitements sylvicoles, aménagement forestier durable, limites d'utilisation des modèles et éthique en modélisation.

Les participants ont ensuite été invités à se projeter dans lavenir et à visualiser quelle place occuperont les différentes approches de modélisation dans dix à quinze ans au Québec. Encore une fois, des approches spécifiques ont été citées de façon prioritaire. Parmi celles-ci, les participants ont mentionné l'importance que prendront les questions liées à la qualité des habitats et des sols dans les futurs modèles de simulation. Parallèlement, ils ont soulevé de façon quasi unanime le virage que devront prendre les prochains calculs de possibilité forestière pour tenir compte de valeurs autres que la matière ligneuse. Ils ont également soulevé l'importance que prendront les modèles par tiges individuelles, les modèles à différentes échelles (écosystème, paysage, régions) et les modèles destinés à mieux prévoir les effets des changements

\footnotetext{
${ }^{7}$ Site du FQRNT : www.fqrnt.gouv.qc.ca.
}

climatiques. La modélisation des effets des risques, la métamodélisation ${ }^{8}$ ainsi que l'intégration des données satellitaires et celles issues de la technologie Lidar occuperont également une place de plus en plus importante.

En dernière partie de cet atelier, les participants ont été appelés à conseiller un jeune diplômé qui en est à ses premières heures en matière de conception d'un modèle de simulation devant servir daide à la décision. Un élément récurrent est ressorti des discussions, soit celui de bien définir l’objectif poursuivi avant de commencer à modéliser un problème particulier et de cerner adéquatement les besoins et le contexte de létude. Certaines personnes ont également jugé important que létudiant puisse identifier un problème simple et puisse létudier en détail. Il a aussi été noté, mais avec moins d’emphase, que létudiant devrait pouvoir faire la différence entre les outils de modélisation qu'il utilisera et la qualité des intrants de son modèle, valider son modèle avec des données fiables, reconnaître les limites de l'approche utilisée et sefforcer d'avoir un modèle doté d'une interface conviviale.

\section{Conclusion}

Ce colloque a mis en évidence le fait que plusieurs modèles de simulation forestière sont utilisés au Québec et a soulevé l'importance de la diversité des modèles. À cet effet, il ressort que les modélisateurs autant que les utilisateurs des modèles devront dorénavant consentir un effort particulier afin de créer des équipes multidisciplinaires et de partager les connaissances en dehors des cercles habituels. De plus, il est important que les besoins de recherche en modélisation forestière soient soutenus par un financement adéquat et continu afin de bien tenir compte des orientations identifiées par les participants. Enfin, il faudra attacher une importance prépondérante à des critères autres que la quantité de bois, tels que la biodiversité, le maintien des cycles des éléments nutritifs du sol ou la capacité de séquestration de carbone des écosystèmes forestiers.

\section{Remerciements}

Les auteurs remercient Mme Marie Anick Liboiron, de Ressources naturelles Canada, pour son aide dans l'organisation de la conférence et l'animation des ateliers de discussion. Nous remercions également Stéphane Tremblay, Josianne Deblois, Pierre Bélanger et Johanne Claveau, du ministère des ressources naturelles et de la Faune du Québec, pour leurs commentaires qui ont permis d'améliorer une première version du manuscrit.

\footnotetext{
${ }^{8}$ La méta-modélisation est une technique de définition des concepts à utiliser pour modéliser des systèmes.
} 